\title{
EOLES COURSE - Five years of remote learning and experimenting
}

\author{
André Fidalgo, Paulo Ferreira e Manuel Gericota \\ Polytechnic of Porto - School of Engineering, Porto Portugal \\ \{anf, pdf, mgg\}@isep.ipp.pt
}

\begin{abstract}
The EOLES (Electronics and Optics e-Learning for Embedded Systems) course consists of a 3rd year Bachelor degree that relies exclusively on e-learning and remote laboratories, developed as the result of an EU funded ERASMUS+ project, involving 15 institutions from four European and three North African countries and concluded in 2015. This paper presents an overview and overall results for this initial period and a more detailed analysis of the Digital Systems Teaching Unit contents, pedagogical approach, grading methodology and results. The focus is on the unit specific characteristics and features, student and teacher experiences and the methodologies that were applied to enhance learning success. The Teaching Unit expositive material is provided as the student progresses, with progressive unlocking of content depending timeline and automatic quizzes results. Grading is divided between weekly assignments, an online exam at the end of each TU and a final exam at the end of the academic year. In short, students are allowed and encouraged to adjust their learning rhythm within the limits allowed by time restraints and evaluation criteria. The developed course was accredited as a specialization year in most partner institutions and has been running non-stop since then, mainly with students from North African institutions. Although no longer supported by an EU project, the course is a good example of sustainability as it already had 4 effective editions with successful approval rates and always with many more candidates than available vacancies.
\end{abstract}

Keywords - E-Learning, Engineering Teaching, Remote Laboratories, Online Course

\section{Introduction}

The degree is EOLES degree is fully delivered on-line using e-Learning 2.0 [1][2] synchronous and asynchronous tools and fully in English language, allowing students to be part of a "virtual learning community" and empowering teamwork, even if the team members are far apart. A dedicated remote laboratory based on virtual experimentation and modelling and simulation platforms, and on remotely operated real instrumentation equipment installed in different universities was used by students to acquire essential practical skills. Degree accreditation is a major advantage of the EOLES course and with particular interest to its main target group. The degree was recognized by the educational authorities of France, Morocco and Tunisia. As a result, all successful students receive a diploma recognized inside the European Higher Education Area (EHEA). 


\section{The EOLES Degree}

\subsection{Organization}

The program was defined in cooperation with the North African Universities participating in the project, considering the priorities defined by their countries' governments. The program's focus on electronics and optics for embedded systems responds to the current tendency for integration of hardware/software into single reconfigurable platforms and to the increase on the amount of data produced and transferred requiring high-speed optical transmission, and to the need of training highly qualified professionals able to keep their countries' pace with these new technologies. The program is divided in fourteen technical units (TUs) and in three optional units. The TUs are divided in two semesters and the detailed content of each one of the TUs is available in the project website [3]. The degree runs for 31 weeks, plus 3 weeks reserved for examinations - one in the end of the first semester, another one in the end of the second semester, and a last one in the final week of the course for make-up exams. The Learning Management System (LMS) that supports TU organization, materials' access and delivery, on-line assessments, virtual and experimental lab access, tracking and reporting, forums and chats and all other course related activities was initially based on a Moodle 2.7 version platform [4], since then upgraded to version 3.5 for the 2018/19 edition

\subsection{Remote Laboratories}

An effective practical innovation of the L3-EOLES degree are the remote laboratories used to perform on-line practical works. A multi-user approach is implemented allowing a group of students to work and interact in real time over the same Practical Work (PW), guaranteeing a strong collaboration among them during the training. Each hardware setup (function generator or oscilloscope, for instance) is connected to the internet. From each TU's Moodle page students have access to the related lab's webpage and to the TUs' proposed lab works. Students can change the hardware configuration in real-time and have an immediate feedback of their actions, via the virtual instrument interfaces that are deployed remotely and through a highdefinition camera (or another interface).

Figure 1 shows one of those lab setups using internet-controlled instrumentation and a camera and part of the user interface. This enables students to see what is going on the real lab and how the real instruments react to their remote commands. This feedback is important for students to be sure that the interface they are seeing in their own monitor is not the visible face of a virtual world but the virtual interface of a real instrument. 


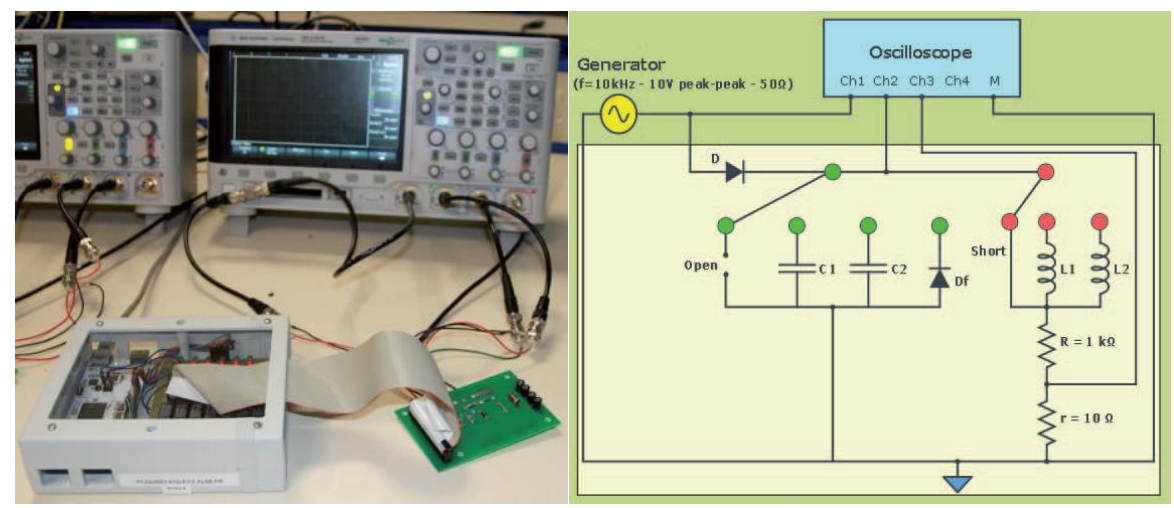

Fig. 1. Remote Laboratory Setup and Interface

The remote laboratory is expected to have a substantial learning impact as each student or group of students have the possibility of repeating the same experiment several times and trying different configurations in a controlled and safe environment. These remote laboratories are used in subjects where the real equipment is more important and were presented and demonstrated on several technical events [5][6]. In some TUs those online labs are replaced or complemented by simulator tools or remote access to advanced software tools.

\subsection{Assessment and Grading}

The EOLES degree follows the French university assessment system with some adaptations. Grading is made on a 0-20 scale, where 10 is the passing grade. Each student is required to have an average of 10.0 or more at the year's end for successful graduation, being possible to have less than 10 on any individual TU, although no grade can be below 5. At the end of the year, there is a final recourse exam, where each student can try to improve his grades on any specific TU in order to achieve passing results. Students that fail to graduate at years end, can repeat only part of the degree (where the failed to achieve a passing grade) on the following year. On each individual TU the grade is composed of three components, namely: (1) mandatory practical works or assignments; (2) an one-hour on-line exam held at the end of each TU; (3) a two-hour final exam held by the semester's end is worth $50 \%$ of the TU's final grade.

The on-line exams are designed to allow the students to consult any technical or pedagogical resource they deem necessary, therefore having a strict time limit and requiring students to be online and visible (through webcam) during the entire exam. The final exam is performed at a university room on a scheduled date, requiring the students to be physically on the same space and under staff supervision during the duration of the exam. A bonus between 0 and 2 points could be attributed at tutor's discretion to each student according to his/her level of participation in the synchronous sessions, forums and live chats. The specific weight of the grading 
components can be adjusted by each TU staff, varying between $20 \%$ and $35 \%$ for components 1 and 2 and $40 \%$ and $50 \%$ for component 3 . The higher weight of the final exam being mostly due to the more controlled environment which provides a fairer grading.

\section{DIGITAL ELECTRONICS TU}

\subsection{Organization}

To better illustrate the degree, we will present and discuss the pedagogical solutions implemented on TU05-Digital Electronics for Embedded Systems. The proposed framework was similar in all TUs, but some implementation adaptations were required as the subjects and difficulty levels are considerably different in some cases. In TU05 the subject is basic digital systems and operations, finite state machines, combinational logic and sequential logic. The lectures consist of a set of 21 pre-recorded asynchronous classes with a duration never exceeding 20 minutes, where an instructor explains the theoretical basis of a subject supported by different types of visual materials as illustrated in Figure 2.

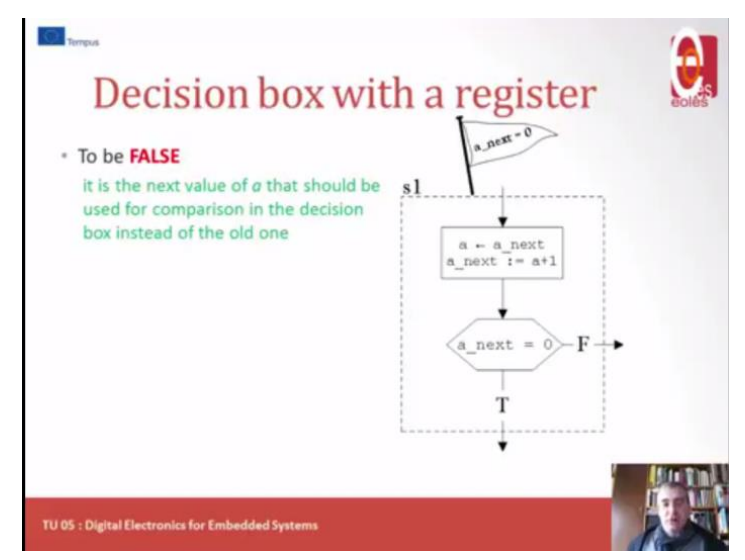

Fig. 2. Synchronous Class

Most classes rely on PowerPoint slides presentations, recorded as online videos, with the teacher image superimposed and several visual aids (arrows, circles, etc...) used to illustrate key points. When required the classes also use external links and access to simulated equipment. The classes are interspersed with self-evaluation quizzes, composed of multiple-choice, fill-in-the-blanks, matching exercises. These are intended to keep students' interest and attention, breaking long expositive classes and have no weight on the TU grading. Additionally, these self-evaluation questions provide students with an immediate feedback about their degree of understanding of the subjects being taught. A Quiz example is presented in Figure 3. 


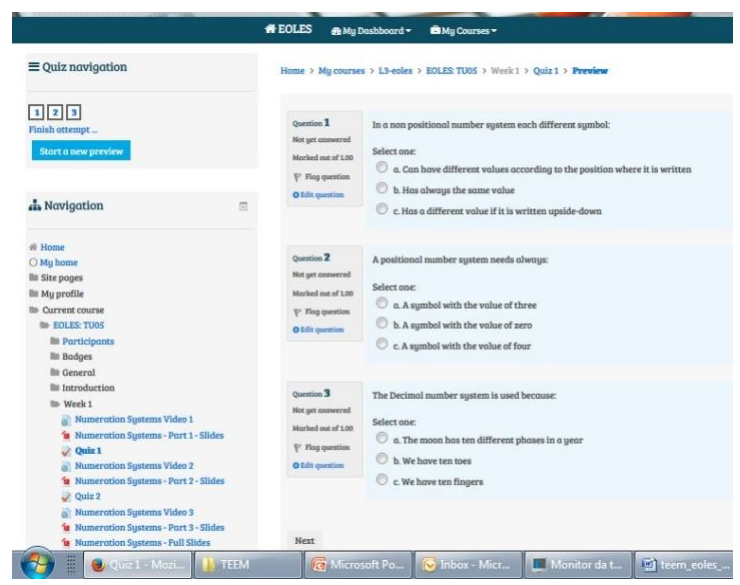

Fig. 3. Self-Assessment Quiz.

Figure 4 presents the initial options available to the students in week 1 of TU05. The first subject is available in both Video and PDF slides formats as well as the first automatic quiz, related to same subject. All other content is unavailable to students and is progressively unlocked as they successfully complete the quizzes. These are not particularly difficult but require the student to pay attention to the video or slides in order to answer the questions. The quiz can be repeated freely and has no effect on final grade, being implemented as a checkpoint.

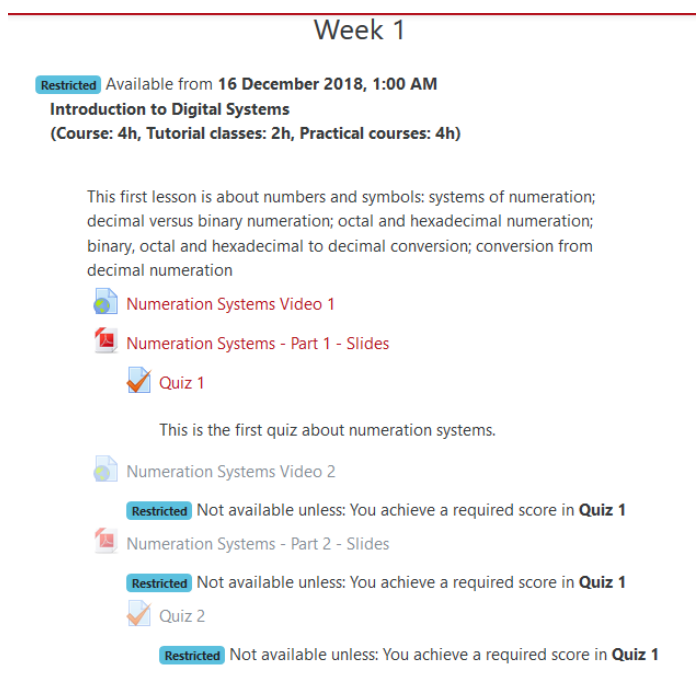

Fig. 4. Week 1 Initial Syllabus 
Students can progress at their own pace, viewing or reviewing this visual material anytime, any number of times, without restrictions. However, the student can only proceed to the next lecture after the successful completion of the self-evaluation questions associated to the previous one. A range of other materials is also available to support the study, including companion books freely downloadable from Internet, web links to other sites containing specialized information and other complementary data, depending on the TUs subject.

Each Week, there is a specific assignment that must be completed by the students and delivered for grading (via moodle). The first week, the assignment is not graded and is based on the successful installation and tutorial completion of the required software for TU05. On subsequent weeks, each student must use the software to solve problems of increasing complexity using the concepts learned on the course. As an expositive example, Figure 5 presents the proposed solutions of parts of two assignments. One is based on the use of Karnaugh Maps to simplify and implement a logical circuit designed to solve a real problem presented in Week 2, and the other is a State Diagram for a different problem presented in Week 4.
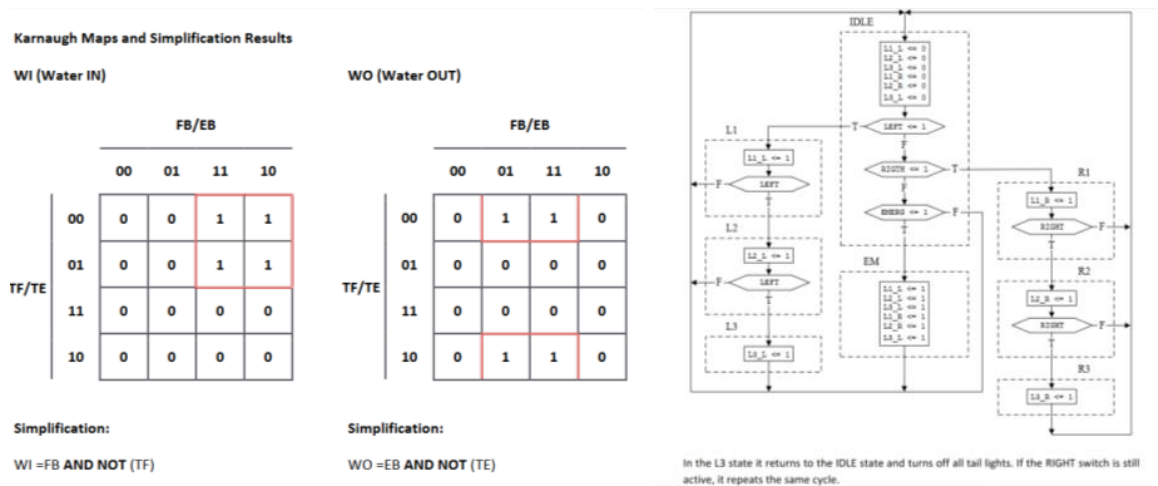

Fig. 5. Assignment Solution Examples

The emphasis in on the application of the learned concepts to practical and operational problems, although simplified due to the remote restrictions. In the case of TU05, all hardware is virtual (VHDL models) and simulated, with the simulation results being used as "proof" of execution. On other TUs, remote laboratories or remote access to software tools are also used to same effect. Each TU uses a different mix of remote labs, simulation and paper exercises depending on technical constraints and course preferences, but all students must do several assignments on the different courses and technologies. Additionally, TU05 relies on tutorial classes which are synchronous classes based on the use of a web conferencing tool. Their aim is to enable students to clarify any issues and ask questions related to the content of the TUs. These classes are also recorded, and the records made available to students. During the synchronous classes tutor and students are required to have their cameras on, with an example being presented in Figure 6. 


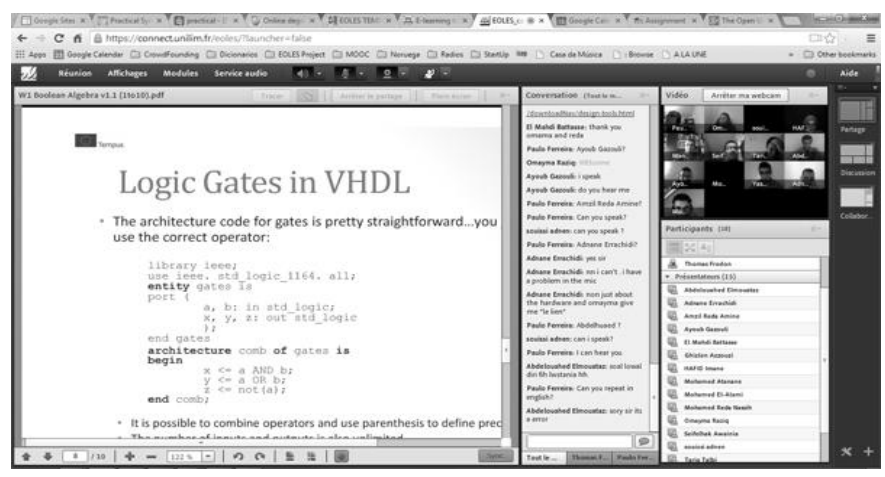

Fig. 6. Synchronous Class.

The aim is to have a visual feedback of the whole class making students feel part of a group and be able to interact not only with the tutor but also among each other.

\section{RESULTS}

\subsection{EOLES Degree}

The number of applicants on all degree editions largely exceeded the expectations, albeit and the number of vacancies were highly concentrated in one of the EOLES partner countries (i.e. Morocco). Table 1 presents the number of students that were enrolled in the degree, those that finished (were present at the final exam) and the approval rates, defined as the number of approved students compared with the number of students that finished the course (attended the exams) or the number originally enrolled, respectively.

\section{Table 1. EOLES Degree Results}

\begin{tabular}{|c|c|c|c|c|c|}
\hline EDITION & ENROLLED & FINISHED & GRADUATED & \multicolumn{2}{|c|}{ APPROVAL } \\
\hline $14 / 15$ & 25 & 21 & 11 & $52 \%$ & $44 \%$ \\
\hline $15 / 16$ & 32 & 26 & 21 & $81 \%$ & $66 \%$ \\
\hline $16 / 17$ & 37 & 34 & 26 & $76 \%$ & $70 \%$ \\
\hline $17 / 18$ & 27 & 22 & 19 & $86 \%$ & $70 \%$ \\
\hline
\end{tabular}

The results are very satisfactory, proving that the issues present in the first edition were sorted and an adequate success rate was achieved. It should be noted the degree is deemed as challenging and requiring effort equivalent to a normal 3rd year degree in a French University, hence the adequacy of the presented success rates. Even so, failure to graduate is always analyzed and three main reasons were identified, namely (1) abandonment of the degree for personal reasons (usually professional); (2) inability to complete some specific TU due to lack of previous knowledge or lack of 
adequate effort; and (3) language problems, albeit having prior English knowledge attested by their TOEFL certificates.

\subsection{Digital Systems Technical Unit}

In the particular case of TU05, the results were in general good, with approval rates of more than $80 \%$ every year and average grades above 12,5 . The online quizzes to get access to content are a key feature of this TU and allowed some specific conclusions. Students would sometimes require several attempts to progress, some had to review the online classes after failing a quiz and others posted their doubts in forums or sent messages to the teachers. These actions were monitored using the Moodle logging functions, and show the quizzes working as intended, promoting interactivity and the need for seeking additional information. The synchronous classes were not used to present new subjects, although several times it was necessary to clarify and repeat issues presented on recorded classes, as some doubts remained. Participation was very variable, with between 25 and $80 \%$ of students present as viewers. However, video and audio participation were limited, with a few students being responsible by the majority of questions and discussion. A very important feature was the ability to share documentation and visual aids, as several questions required the discussion and revision of available materials. The final online exam had excellent results, the grade distribution being presented in Figure 7.

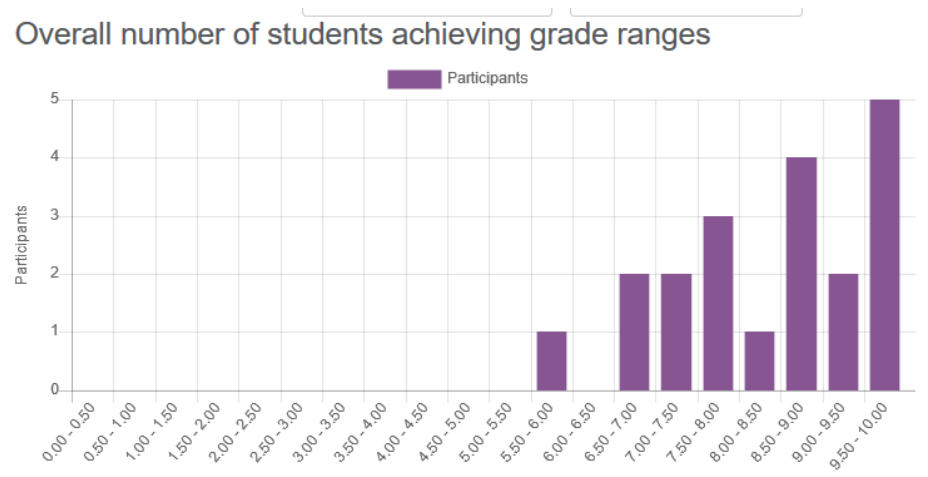

Fig. 7. Synchronous Class.

The practical assignments were a mixed experience. In TU05 the initial assignments were simpler and direct, with most assignment providing the student with a good grade. In the last week the assignment was more complex and was more frequent that students would not even attempt it (with the negative effect on the final grade) and several others being obviously incomplete attempts or rushed by the students. In all assignments there was also some need to analyze the uniqueness of the delivered reports, as the online character of the course sometimes promoted the sharing of results between students. The online exams usually present much better 
grades that the final exams, as was to be expected, and showing that some type of attendant assessment is still required for a fairer grading.

In this TU, most grades were above the 50\% mark, confirming that the TU subjects were adequately delivered. The examination results were better than assignment work results, by a considerable margin in several cases, due to somewhat tough learning curve of the high-end software being used on assignments [7]. Nevertheless, this experimental work is deemed as a vital learning resource and every year the documentation and support are improved.

\section{CONCLUSIONS}

EOLES is an excellent example of sustainability, providing an effective and selfsustained online degree with continuous operation and successful editions. One of its main strengths derives from the its formal accreditation on several countries that provides the main motivation for annual student applications. As all online and traditional courses, the longevity required it to provide the necessary tools and knowledge to allow students to succeed into a very specialized labor market, and that success to assist in the degree continuous evolution.

As to the effectiveness of the learning environment, several non-quantitative conclusions were possible from the first years' experience, namely: (1) Students are more used to interactive classes, preferring those as an initial approach. This solution is feasible for simpler subjects; (2) Recorded classes are a much more time efficient way to deliver complex subjects, as they allow the students to study and repeat at their own pace; (3) Interactive classes are required to clarify doubts and answer questions. The use of recorded classes is not a complete solution to most students; (4) Student participation in interactive classes is very diverse, requiring the teacher to be proactive; (5) Additional asynchronous resources (forums, emails) are often preferred by some students, namely when lacking communication skills (e.g. English language)

The overall experience of the teaching staff is extremely positive and allows for a permanent improvement of the teaching process. There are new challenges on each edition, and it is necessary to keep improving and evolving in order to keep up with the technological advances and the student's expectations.

The experience gained from the development of this degree is being used as a base of an ongoing ERASMUS+ project [8], where it is intended to develop a set of practices and methodologies to be used in the implementation of other online courses and laboratories in general, but with particular attention to engineering degrees. 


\section{REFERENCES}

1. Andre Fidalgo et al., "The EOLES project - Engineering labs anywhere", Proceedings of the IEEE Global Engineering Education Conference (EDUCON'2014), April 2014, pp. 943-946

2. T. Bates, "Understanding web 2.0 and its implications for E-learning", in M. J. W. Lee, and C. McLoughlin (Eds), Web 2.0- Based E-learning: Applying Social Informatics for Tertiary Teaching, IGI Global, New York, pp. 21-42, 2011

3. "Course Content", EOLES project Website, Internet: www.eoles.eu [September 2019]

4. "Moodle," Internet: moodle.org [Apr 2019]

5. S. Farah, A. Benachenhou, G. Neveux, D. Barataud, G. Andrieu, T. Fredon, "Flexible and Real-Time Remote Laboratory Architecture Based on Node.js Server", 3rd Experiment@International Conference (exp.at15), Pont Delgada, Azores, Portugal, June 2015

6. S. Farah, A. Benachenhou, G. Neveux, D. Barataud, "Design of a Flexible Hardware Interface for Multiple Remote Electronic Practical Experiments of Virtual Laboratory", International Journal of Online Engineering, Vol. 8, Special Issue 2, March 2012, pp. 7-12

7. www.xilinx.com/products/design-tools/vivado.html [April 2019]

8. M. Gericota, P. Ferreira, A. Fidalgo, G. Andrieu, C. Dalmay, The e-LIVES Project: eEngineering Where and When Students Need, 2019 IEEE Global Engineering Education Conference (EDUCON), Dubai, UAE, April 2019 